Cahiers d'études italiennes

\title{
Corrado Stajano, écrivain étranger à son passé
}

\section{Claudio Milanesi}

\section{OpenEdition \\ Journals}

Édition électronique

URL : http://journals.openedition.org/cei/286

DOI : $10.4000 /$ cei.286

ISSN : 2260-779X

\section{Éditeur}

UGA Éditions/Université Grenoble Alpes

\section{Édition imprimée}

Date de publication : 15 juin 2005

Pagination : 181-192

ISBN : 978-2-84310-066-6

ISSN : $1770-9571$

Référence électronique

Claudio Milanesi, «Corrado Stajano, écrivain étranger à son passé », Cahiers d'études italiennes [En ligne], 3 | 2005, mis en ligne le 15 décembre 2006, consulté le 27 mars 2021. URL : http:// journals.openedition.org/cei/286 ; DOI : https://doi.org/10.4000/cei.286 


\title{
CORRADO STAJANO
}

\author{
ÉCRIVAIN ÉTRANGER À SON PASSÉ
}

\section{Claudio Milanesi}

Université de Provence - Aix-Marseille 1

Journaliste, intellectuel, sénateur de la République de 1994 à 1996, Corrado Stajano est l'auteur d'une dizaine de livres sur les grandes questions qui ont nourri le débat politique et intellectuel italien de la fin des années 1970 à nos jours. Avec Giorgio Bocca, Enrico Deaglio, Gianni Barbacetto, Giorgio Boatti, Stajano est l'un des auteurs de référence d'un genre d'écriture de la réalité qui se situe au croisement du journalisme, de l'histoire du présent et de la littérature. Dans chacun de ses livres, Stajano a adopté un registre différent : livre de souvenirs, journal, échange épistolaire, biographie, etc. Ses livres ont le mérite d'avoir fait évoluer les codes de l'écriture de la réalité en adoptant des solutions narratives et stylistiques originales et élaborées. Quant aux thématiques qui y sont abordées, certaines sont consacrées aux phénomènes de violence collective, notamment aux mouvements sociaux des années 1970 (Il sovversivo), au terrorisme (L'Italia nichilista) et, en remontant dans le temps, à la Résistance et à la période de la guerre civile des années 1943-1945 (Patrie smarrite) ${ }^{1}$.

1. Corrado Stajano, (avec Giampaolo Pansa), Le bombe di Milano, Parme, Guanda, 1970 ; Corrado Stajano, La pratica della libertà, Turin, Einaudi 1976; (avec Marco Fini), La forza della democrazia, id., 1977 ; Il sovversivo. Vita e morte dell'anarchico Serantini, id., 1975 (2éd., id., 1992) ; Africo. Una cronaca italiana di governanti e governati, di mafia, di potere e di lotta, id., 1977 ; (avec Giovanni Russo), Terremoto : le 2 Italie sulle macerie del Sud : volontari e vittime, camorristi e disoccupati, notabili e razzisti, borghesi e contadini, emigrati e senzatetto, Milan, Garzanti 1981 ; L'Italia nichilista. Il caso di Marco Donat Cattin, la rivolta, il potere, Milan, Mondadori, 1982 (2e éd., Turin, Einaudi 1992) ; (avec Giovanna Borgese), Un paese in tribunale, Milan, Mondadori 1983 ; Mafia. L'atto di accusa dei giudici di Palermo, Rome, Editori Riuniti, 1986 ; Un eroe borghese, Turin, Einaudi, 1991 ; Il disordine, id., 1993 ; Promemoria. Uno straniero in patria tra Campo de' Fiori e palazzo Madama, Milan, Garzanti, 1997 ; (avec Gherardo Colombo), Ameni inganni, id., 2000 ; Patrie smarrite. Racconto di un italiano, id., 2001. 
L'œuvre de Stajano couvre donc toute la période qui va de la Seconde Guerre mondiale à nos jours, et s'intéresse notamment aux deux explosions de violence collective qui ont marqué l'histoire italienne récente : d'une part, la guerre civile qui, dans la péninsule, se déroula parallèlement aux événements de la Seconde Guerre mondiale ; d'autre part, ce qu'on a appelé la strategia della tensione et les années de plomb, c'est-à-dire l'époque qu'on situe généralement entre l'attentat de Piazza Fontana, le 12 décembre 1969, et l'année 1983, l'année des premiers grands procès contre les membres des organisations terroristes des Brigades rouges et de Prima Linea.

Avec le recul dont nous bénéficions aujourd'hui, nous pouvons nous demander s'il est possible de trouver des liens, des lignes de continuité entre ces deux séries d'événements que sépare l'espace d'une génération. La preuve en est ce colloque même, dont l'intitulé suggère déjà que leur parenté est constituée par le recours à la violence. Déjà, dans les années 1970 , on pensait à cette continuité entre les deux séries d'événements. On l'expliquait par la continuité des modes d'action entre le fascisme des années 1940 et les groupes néo-fascistes à l'origine du terrorisme d'État, de Piazza Fontana à l'attentat de la gare de Bologne ; et, au sein même d'un courant des Brigades rouges, on invoquait une sorte de continuité mythique entre la lutte antifasciste de la Résistance et la prétendue Nouvelle Résistance des années 1970. Renato Curcio et Alberto Franceschini, qui sont parmi les fondateurs des Brigades rouges, ont à plusieurs reprises évoqué une sorte de rite d'initiation au cours duquel les anciens partisans de la lutte antifasciste passaient les armes qui étaient restées cachées pendant vingt ans aux mains des nouveaux résistants de la lutte armée ${ }^{2}$. Et Corrado Stajano de rappeler qu’à Turin, en 1976, le groupe de Marco Donat Cattin, qui plus tard donnera naissance à Prima Linea, signe Senza tregua ses premiers attentats, en se rattachant de façon explicite à l'expérience de la guérilla urbaine des GAP, les Groupes d'action partisane actifs de 1943 à 1945 dans l'Italie occupée par les Allemands ${ }^{3}$.

Désormais, c'est sous un autre jour que l'on s'interroge sur les éléments de continuité entre la guerre civile des années 1940 et le terrorisme. Le

2. Voir par exemple Renato Curcio (avec Mario Scialoja), A viso aperto, Milan, Mondadori, 1993, p. 71. Le journaliste anglais Tobias Jones, dans un excellent chapitre consacré au terrorisme italien ( $\mathrm{La}$ madre di tutte le stragi), évoque les événements de ces années ainsi que ces deux lignes de continuité : Tobias Jones, Il cuore oscuro dell'Italia, Milan, Rizzoli, 2003, p. 51-88 (éd. angl. The dark heart of Italy, 2003).

3. Voir Stajano, L'Italia nichilista, éd. 1992, op. cit., p. 204-205. 
dernier film de Marco Bellocchio, Buongiorno notte (2003), qui réinterprète l'enlèvement et le meurtre du président de la DC Aldo Moro par les Brigades rouges (16 mars-9 mai 1978), est à cet égard instructif, puisqu'il montre en parallèle le sort tragique du président de la Démocratie chrétienne, exécuté de sang-froid dans un garage de la banlieue de la capitale, et celui des condamnés à mort de la Résistance. La continuité s'appuie donc sur le meurtre, sur la mort donnée par un pouvoir qui s'arroge le droit de disposer de la vie des hommes. De ce point de vue, dans l'œuvre de Stajano, on ne trouve jamais l'évocation de ces deux types de parallélisme. Ses ouvrages sur le terrorisme et la guerre civile restent relativement indépendants les uns des autres. Et ce n'est qu'indirectement que se font jour les liens entre les deux phénomènes.

\section{Patrie smarrite}

Le premier des deux livres que nous allons analyser est le journal Patrie smarrite, publié en 2001. Ce texte a, à la fois, les caractéristiques d'un journal de voyage, d'un journal intime et d'un livre de souvenirs personnels et collectifs. Dans la lignée des romans-essais tels que Danubio de Claudio Magris, Patrie smarrite remonte dans le temps et dans l'inconscient des deux villes natales des parents de Stajano, Noto et Crémone. C'est un voyage réel et concret, narrant des rencontres avec des témoins de la Seconde Guerre mondiale, et c'est aussi un parcours dans l'imaginaire, à travers les marques que le temps a laissées dans les archives, dans l'urbanisme, ainsi que dans certains des écrits et comptes rendus des grands événements qui ont marqué l'histoire des deux villes. À tout cela, s'ajoute la mémoire individuelle de l'enfance du narrateur Stajano.

L'occasion à la base de la rédaction de cette enquête est double : d'une part, l'arrivée au gouvernement, en 1994 puis en 2001, d'une coalition qui - qu'on le veuille ou non - suscite une certaine appréhension dans une partie de l'opinion à cause de la présence en son sein de formations et de courants politiques directement ou indirectement liés aux années de la dictature ; d'autre part, le débat suscité par le révisionnisme appliqué à l'histoire de la Seconde Guerre mondiale en Italie. C'est en 1996 que, publiant son livre Morte della Patria, le journaliste et historien Ernesto Galli della Loggia provoque un vaste débat sur le rôle et le sens de l'antifascisme dans l'histoire de l'Italie contemporaine. Galli della Loggia prétend que le vide laissé par ce qu'il appelle la mort de la patrie, c'est-à-dire l'effondrement militaire et politique de l'armée et de l'État qui eut lieu le 
8 septembre 1943, n’a jamais été comblé par les valeurs de l'antifascisme et de la République. Il estime que, avec la fin de la guerre froide, le temps est venu d'insérer l'expérience de la dictature et du fascisme dans l'histoire $\mathrm{du}$ pays. Une véritable renaissance de la patrie ne serait possible qu'en abandonnant les prétentions à l'exclusivité des valeurs de l'antifascisme, car, selon Galli della Loggia, elles ne seraient pas les valeurs de toute la patrie, mais seulement d'une partie de la communauté nationale ${ }^{4}$.

De la part de cet intellectuel qui se déclare libéral, cette provocation était en fait une tentative, une opération culturelle et politique visant à légitimer l'arrivée au pouvoir du parti des héritiers du fascisme, Alleanza Nazionale, et visant à discréditer, en niant sa légitimité, ce que l'on appelait " l'arc constitutionnel ", c'est-à-dire l'ensemble des partis des forces antifascistes ayant fondé et marqué la vie politique de la République de 1946 à 1992. À l'époque de la publication du livre de Galli della Loggia, le seul parti survivant était désormais le Parti démocratique de gauche, héritier de l'ancien Parti communiste italien. Sur le plan historique, cela revenait à dire, dans le sillage de l'historien du fascisme Renzo De Felice, que la balance était égale entre fascisme et antifascisme, entre le choix des jeunes gens ayant décidé de s'enrôler dans l'armée de la République de Salò alliée à l'Allemagne nazie, et celui des jeunes gens du même âge ayant combattu le nazisme et le fascisme dans les rangs de la Résistance. Le débat fit rage, et il continue encore dans les journaux et sur le terrain de la mémoire collective, constituant ainsi un véritable enjeu politique : tel président de région prétend réécrire les livres d'histoire en introduisant un jugement " équitable » sur le fascisme et l'antifascisme, tel maire réinstalle dans la galerie de portraits de sa mairie celui d'un prédécesseur collaborateur, etc.

C'est dans ce débat, à la fois historiographique, culturel et politique, qu'il convient de placer le livre de Stajano : les deux séjours dans les villes natales de ses parents sont l'occasion d'une recherche et d'une interrogation sur l'attitude de la population de ces deux villes lors de l'effondrement du 8 septembre. Les deux villes se trouvant l'une en Sicile, l'autre dans la plaine du Pô, elles durent faire face à deux issues différentes de la

4. Ernesto Galli della Loggia, La morte della Patria. La crisi dell'idea di nazione tre Resistenza, antifascismo e Repubblica, Rome/Bari, Laterza, 1996. La comparaison entre ce texte de Galli della Loggia et les deux ouvrages de Elena Aga Rossi, Una nazione allo sbando. L'armistizio italiano del settembre 1943, Il Mulino, Bologne, 1993 et de Claudio Pavone, Una guerra civile. Saggio storico sulla moralità della Resistenza, Turin, Bollati Boringhieri, 1991, nous aidera à comprendre la différence entre la révision, nécessaire et toujours renouvelée, de l'histoire nationale, et le révisionnisme historique, dont le texte de Galli della Loggia est un exemple parlant. 
défaite militaire, laquelle se situe entre le débarquement des forces alliées en Sicile (10 juillet 1943), la démission et l'incarcération de Mussolini (25 juillet), l'armistice avec les forces alliées et l'invasion de l'armée allemande (8 septembre). Noto fut en effet occupée par les Alliés dès le début du mois de juillet, alors que Crémone, fief d'un des dirigeants les plus inflexibles et extrémistes du régime, Roberto Farinacci, fut témoin de l'effondrement du pouvoir fasciste et de la fuite de Farinacci, qui s'exila auprès de Hitler à Berlin, avant de revenir après l'invasion allemande et la naissance de la République sociale, en septembre 1943.

Le propos de Stajano n'est pas simple à déchiffrer, car le livre est une sorte de journal intime accompagné de recherches dans la mémoire collective, qui suscite à son tour la mémoire individuelle, et non pas une recherche historique défendant une thèse bien définie. Le travail de mémoire est douloureux car il dépasse les idées reçues, les a priori, les choix idéologiques, et fait surtout remonter à la surface ce qu'il y a de désagréable et de troublant, car il est contradictoire et dérangeant pour chacun, pour l'ego et pour l'image qu'on s'est construits de soi-même et de sa patrie. Stajano essaie d'éviter la tentative révisionniste de mettre sur le même plan les défenseurs de la liberté et les alliés de la barbarie, la Résistance et les "ragazzi di Salò ». Il essaie également d'éviter la rhétorique simpliste d'un certain antifascisme institutionnel, pour qui le fascisme ne fut qu'une parenthèse et les fascistes une sorte de martiens venus d'ailleurs, en se contentant de l'évocation des valeurs de la Résistance pour absoudre les complicités et les faiblesses de la société italienne face à la dictature. Stajano part donc du refus des deux versions de ce que Silvana Patriarca a qualifié de néo-patriotisme italien ${ }^{5}$ : le néo-patriotisme de la droite qui, à travers la révision du fascisme, songe à sa réhabilitation, et le néo-patriotisme de gauche (qu'incarne l'actuel président de la république Carlo Azeglio Ciampi), qui se satisfait du rappel aux valeurs du Risorgimento, de la Résistance et de la République - comme si l'histoire de l'Italie était une sorte de réalisation progressive des libertés interrompue accidentellement par la montée du fascisme en 1922, ou par les victoires aux élections de 1994 et de 2001de la nouvelle droite réunie dans la coalition de la "Maison des libertés».

5. Voir Silvana Patriarca, Italian neopatriotism : "Debating national identity in the 1990s ", Modern Italy, VI, 1, 2001, p. 21-34. Sur la place de Stajano dans le débat sur le néo-patriotisme italien des années 1990, voir Claudio Milanesi, "Patria senza, patrie smarrite, nuove idee di patria : neopatriottismo e libertà nella cultura italiana alla fine della Prima Repubblica ", Italies. Littérature Civilisation Société, 6, 2002, p. 339-350. 
Stajano explore les zones sombres des lâchetés et des compromissions de la bourgeoisie italienne, les comportements troubles et l'attitude ambiguë de la classe dirigeante. En Sicile, par la juxtaposition des bulletins de guerre de l'armée, des comptes rendus des événements de juillet 1943 rédigés par les notables de la ville de Noto, des messages que s'échangeaient les autorités, des gros titres de la presse du régime et des discours de Mussolini (le "discorso del bagnasciuga ${ }^{6}$ "), Stajano laisse le lecteur découvrir lui-même qu'en fait, en dépit de la rhétorique tonitruante du régime, des bataillons entiers de l'armée italienne ont renoncé - par opportunisme ? - à combattre l'invasion alliée. L'armée anglaise pénétra à l'intérieur de l'île sans rencontrer de véritable résistance. Churchill remarque avec ironie que, lors de la prise de Lampedusa et de Pantelleria, le seul soldat blessé de l'armée anglaise avait été mordu par un âne. Les Anglais entrent à Noto dans un silence presque irréel, et le bruit lointain qui les inquiète pendant quelques instants n'est en fait que celui d'une procession improvisée par les autorités religieuses venant leur remettre les clés de la ville. Les soldats de l'armée italienne - ceux-là mêmes dont le journaliste Mario Appelius louait dans les colonnes du Popolo d'Italia le " calme viril et la confiance chaleureuse ${ }^{7}$ » - ont disparu, troquant pendant la nuit leur uniforme contre des vêtements civils. Et la liste des lâchetés s'allonge : tel bataillon hisse le drapeau blanc avant même d'en avoir reçu l'ordre, tel pilote de chasse communique avoir détruit deux croiseurs alors qu'il est rentré à la base en prétextant une panne de carburant, tel général parvient à quitter indemne l'aéroport qu'il devait défendre car il a vraisemblablement troqué sa liberté contre l'abandon à l'ennemi d'installations intactes, etc.

À Crémone, c'est peut-être encore pire : la ville, qui avait été le creuset des coopératives agricoles au début du siècle, se livre au fascisme dès le mois d'octobre 1922. Certes, la nuit du 27 octobre, l'armée résiste à l'assaut que les squadre d'azione de Farinacci donnent à la préfecture, et réussit à repousser les assaillants. Mais, dès le matin du 28, quand se répand la nouvelle de la désignation de Mussolini, les fascistes peuvent enfin se montrer au balcon de la préfecture, aux côtés des autorités de l'État. Le préfet de police écrit alors ce télégramme au ministère : " città è festante ed unanime nel suo compiacimento per imponente manifestazione fascista »

6. On lira le texte du discours de Benito Mussolini publié dans Il popolo d'Italia le 5 juillet 1943 en annexe de Renzo De Felice, Mussolini l'alleato, Rome/Bari, Laterza, p. 1466-1479 (puis Turin, Einaudi, 1990).

7. Mario Appelius, dans Il popolo d'Italia du 14 juillet 1943. Cité par Stajano, Patrie smarrite, op. cit., p. 56. 
(la ville est en liesse et unanime dans son accueil favorable à l'imposante manifestation fasciste) ${ }^{8}$. Dès lors, c'est le crescendo du consensus, que Stajano redécouvre à travers les photographies de la vie quotidienne de Crémone dans les années 1930 : les rues qui regorgent de gens à l'arrivée du duce ou du roi, les avant-gardistes soufflant dans leurs trompettes, les parades des faisceaux féminins, les « fils de la louve » qui montent la garde devant l'or recueilli pour la patrie... Toute la ville paraît cultiver la flatterie du pouvoir et la servilité abjecte. Quand enfin, le 25 juillet 1943, Mussolini est arrêté et que Farinacci doit chercher refuge en Allemagne, «à Crémone il ne se passe rien" (ibid., p. 169) : l'ordre règne, les antifascistes sont évanescents, le journal fasciste est repris par un directeur modéré, la bourgeoisie d'une ville qui s'est tellement compromise avec la dictature est incapable de se racheter. Deux ans plus tard, à la Libération, Farinacci, revenu entre-temps, s'enfuit à nouveau, et ce n'est pas à Crémone mais à Vimercate, près de Milan, qu'il sera jugé et exécuté. Quand Stajano, plus de cinquante ans après, essaiera de retrouver le souvenir de cette compromission de la ville avec le régime, il se heurtera à un mur d'oubli et d'indifférence.

Cela devrait suffire à comprendre que Patrie smarrite n'entre pas dans la catégorie de la littérature hagiographique de la Résistance. Ce n'est pas non plus un livre révisionniste dans le sens idéologique qu'a pris ce terme dans le débat actuel. Malgré tout, pour Stajano, la différence entre ceux qui ont combattu pour la liberté et la justice et ceux qui ont « tout fait pour l'étouffer " (ibid., p. 127) est claire et définitive. Stajano ne tombe pas non plus dans le piège qui consiste à dire que tout le monde étant complice, tout le monde est finalement innocent. Non, en ces moments de crise morale, ce sont les vices constants de l'histoire italienne sur le long terme, que Stajano paraît presque considérer comme des caractères nationaux - la lâcheté des habitants de Noto et l'onctuosité mielleuse des Crémonais n'étant que des métaphores de ceux-ci -, qui remontent à la surface. La cible de Stajano est la bourgeoisie italienne, qui paraît toujours prête à sacrifier la liberté pour conserver ses privilèges et défendre ses intérêts, du temps de Farinacci à Crémone ou de nos jours devant la victoire électorale de la coalition de centre droit.

Dans ces conditions, pour Stajano aussi, la patrie serait-elle morte le 8 septembre ? Absolument pas : la patrie est morte bien avant, avec les compromissions de la bourgeoisie extrémiste de Crémone, avec la vulga-

8. Cité par Corrado Stajano, ibid., p. 138. 
rité et la violence de son Ras Farinacci, avec le ralliement de toute une société à la dictature. Et, malgré la victoire finale des forces antifascistes, la honte du compromis est telle que le sentiment d'attachement à la patrie en sort blessé, perdu, "smarrito ", non pas tellement à cause du 8 septembre, mais à cause d'une longue habitude à la lâcheté et à l'opportunisme des classes dirigeantes italiennes. Pour Stajano, le résultat est cet intense sentiment d'être un étranger dans son pays, d'être tellement loin de l'histoire de ses racines, et donc de sa propre histoire, qu'il en devient comme étranger à lui-même. Straniero in patria : c'était justement le soustitre de son ouvrage précédent, Promemoria, le journal de son expérience romaine vécue en qualité de sénateur de "l'alliance des progressistes ", de 1994 à 1996.

\section{L'Italia nichilista}

L'autre livre de Stajano qui a retenu notre attention est L'Italia nichilista. Il caso di Marco Donat Cattin, la rivolta, il potere, publié pour la première fois en 1982, vingt ans avant Patrie smarrite. Nous nous demanderons plus loin s'il existe un lien entre les deux. Ce livre est une vraie perle rare dans l'immense bibliographie sur le terrorisme italien des années 1970. Biographies, autobiographies, interviews, témoignages, enquêtes, romans, poèmes, pamphlets : dans ce vaste ensemble d'ouvrages qui constituent désormais presque un genre en soi, le livre de Stajano brille par son inspiration, par la dignité et la clarté de son engagement en faveur de la vérité et de la justice, par les principes de sa composition, par la finesse du montage des matériaux narratifs, par la richesse de son analyse et la multiplicité des points de vue et des voix qui y apparaissent. En 1982, Stajano était déjà l'auteur de deux livres : l'un, Il sovversivo, sur Franco Serantini (un jeune manifestant, mort des suites d'un passage à tabac par les forces de l'ordre lors d'une manifestation à Pise, en 1972), l'autre, Africo, très différent, une enquête sur l'éternel retour de ce que l'on a appelé pendant longtemps la questione meridionale, vue à travers la vie de la commune calabraise d'Africo. Stajano avait pu ainsi affiner son style et élaborer son modèle d'un nouveau genre, au croisement du journalisme, de la microhistoire, du pamphlet et de la narration.

Ce livre de Stajano n'est donc pas une œuvre de fiction mais le résultat d'une recherche fort documentée, utilisant toutefois l'ensemble de la panoplie des procédés littéraires de fictionalisation : montage de documents ; citation de témoignages; imbrication de plans temporels de la 
narration ; analyse de faits juxtaposés, partant de la vie privée pour arriver à la politique nationale, en passant par le plan local et le domaine judiciaire. L'Italia nichilista est en fait la double biographie de Carlo et Marco Donat Cattin : le premier, cacique de la Démocratie chrétienne et ministre du travail dans plusieurs gouvernements successifs, mort en 1991 ; le second, son fils, d'abord militant des mouvements contestataires au début des années 1970, puis, dès 1976, dirigeant et membre actif du groupe terroriste Prima Linea, et enfin « repenti » et collaborateur de la justice mort le 19 juin 1988 dans un accident de la route.

Ce livre aurait pu n'être qu'une enquête et un témoignage parmi les dizaines qui ont été publiées sur le terrorisme et qui ont encombré les rayons des librairies'. Mais il ressort nettement du lot pour plusieurs raisons. Loin de se cantonner dans une attitude de neutralité teintée d'une volonté de comprendre, et finalement d'absoudre, la génération de la lutte armée au nom de l'injustice qu'elle prétendait combattre par des moyens tels que les gambizzazioni ${ }^{10}$ ou le meurtre, Stajano ne renonce pas aux jugements de valeur tout en sauvegardant la précision et la richesse de sa documentation. La clé du livre est dans la mise en parallèle des parcours du fils et du père. D'après l'auteur, rien ne peut justifier les méthodes des groupes terroristes de cette époque : Stajano stigmatise l'irresponsabilité, l'esthétisme complaisant, le narcissisme de ses membres. Il croise le récit des actions politiques avec celui de la vie privée de ces anciens militants contestataires qui glissent, sans solution de continuité, dans la clandestinité et le terrorisme. C'est là que le lecteur découvre la double vie de ce dirigeant et membre fondateur de Prima Linea, la deuxième formation terroriste italienne après les Brigades rouges : assassin la veille, et le lendemain en vacances dans les localités les plus « branchées » de la Sardaigne ou du Val d'Aoste. C'est là aussi que l'on découvre l'attitude irresponsable et opportuniste d'un père, qui se mobilise pour retrouver et faciliter la fuite

9. Les membres des Brigades rouges se sont distingués par la production de témoignages et d'interviews : voir par exemple Renato Curcio (avec Mario Scialoja), A viso aperto, Milan, Mondadori, 1993 ; Mario Moretti (avec Rossana Rossanda et Carla Mosca), Brigate rosse. Una storia italiana, Milan, Baldini \& Castoldi, 2002 (1ère éd. Anabasi, 1994); ou Anna Laura Braghetti (avec Paola Tavella), Il prigioniero, Milan, Feltrinelli, 2003 ; six autres biographies de membres des Brigades Rouges ont été recueillies par Giovanni Bianconi, Mi dichiaro progioniero politico, Turin, Einaudi, 2002 ; les adhérents à Prima Linea ont été beaucoup plus discrets. Sur le phénomène du terrorisme rouge on peut utilement consulter la recherche du Progetto memoria, La mappa perduta, Rome, Sensibili alle foglie, 1994 ; plus généralement, sur la strategia della tensione et les années de plomb, voir le bilan du président de la Commission parlementaire d'enquête sur le terrorisme en Italie, Giovanni Pellegrino (avec Giovanni Fasanella et Claudio Sestieri), Segreto di Stato. La verità da Gladio al caso Moro, Turin, Einaudi, 2000.

10. La victime d'une gambizzazione n'était ni séquestrée ni tuée, mais blessée aux jambes par des coups de feu. Ce fut le cas du doyen du journalisme italien, le conservateur Indro Montanelli, blessé aux jambes le 2 juin 1977, à Milan, par un commando des Brigades rouges. 
de son fils seulement quand il voit son propre pouvoir menacé par les révélations d'un terroriste repenti, qui a dévoilé aux carabiniers que le fils du ministre Donat Cattin fait partie d'une bande armée.

L'histoire de la lutte armée devient alors une autre histoire typiquement italienne, histoire faite de compromis, d'opportunisme, de lâchetés, de servilité ; et aussi de "familialisme amoral ", de corruption, d'absence d'éthique au sein de la classe politique ; fautes et faiblesses de la classe dirigeante, qui engendrent une génération de terroristes privilégiés et protégés, terroristes les jours ouvrables et le week-end à la montagne. Quand on l'observe dans son déroulement quotidien, la vie de Marco Donat Cattin et de ses complices perd toute aura romantique : Roberto Sandalo, son meilleur ami, lui aussi terroriste et lui aussi " repenti ", ne trouve un emploi chez une concessionnaire d'automobiles que grâce à la recommandation du ministre Donat Cattin ; Marco lui-même, secrétaire au lycée Ferraris de Turin, demande un congé d'un semestre pour justifier ses absences répétées, en prétextant la difficulté de sa vie de père célibataire. Mais s'il renonce à aller travailler, Marco ne renonce jamais à ses vacances de fils à papa : en juillet 1979, par exemple, juste après avoir participé à l'exécution de Carmine Civitate, le tenant du bar dell'Angelo, soupçonné par l'organisation Prima Linea d'avoir signalé à la police la présence de deux de ses membres dans son établissement ${ }^{11}$, Donat Cattin part pour des vacances de rêve, que peu de jeunes gens de son âge pouvaient se permettre à l'époque : en Sardaigne, sur le bateau d'une famille amie de ses parents, dans l'île de Alicudi ; puis un court séjour à Rome, et quelques jours dans un chalet dans la vallée d'Aoste.

Peut-on considérer cette génération, passée des rêves de liberté de 1968 au cauchemar de la lutte armée, comme responsable elle-même de ses propres erreurs et déviations? Ou bien, les fautes des pères excusent-elles celles de leurs enfants? Inversement, en quoi les erreurs d'un fils doiventelles retomber sur son père ? Pour Stajano, les deux générations partagent la responsabilité, et ce partage n'atténue en rien la portée de leurs erreurs. D'abord, parce que la liste des griefs envers Carlo Donat Cattin, métonymie de la classe dirigeante italienne au même titre que son fils est celle de la génération d'innocents à laquelle il appartient, est longue : quand Marco, à l'âge de dix-huit ans, est mis en garde à vue pour agression contre des

11. D'après certaines sources, Civitate avait en effet appelé la police à cause du comportement violent de Matteo Caggegi : ce dernier et Barbara Azzaroni seront tués par la police dans l'échange de coups de feu qui suivra à leur interpellation le 28 février 1979. Cf. "Progetto memoria ", La mappa perduta, p. 301 et 429 . 
jeunes d'extrême droite, il parait bénéficier d'une sorte d'invisible filet de protection. Est-ce son père qui intervient directement pour le protéger? $\mathrm{Ou}$ pis, la protection se déclenche-t-elle automatiquement parmi les forces de l'ordre et la Justice, à cause de leur servilité à l'égard du ministre démocrate-chrétien ? Plus tard, quand commence à courir le bruit de la participation de Marco à des attentats, Carlo Donat Cattin va voir le président du Conseil et futur président de la République, Francesco Cossiga, afin de lui demander des informations, et peut-être une protection, pour son fils. On ne saura jamais vraiment ce qui s'est passé dans le secret du Palazzo Chigi. Mais ce qu'on sait, quoi qu'il en soit, c'est que le fils, peu de temps après, trouvera refuge à Paris, et que le président du Conseil sortira blanchi du scandale provoqué par cette confusion d'intérêts familiaux et politiques. Et Carlo Donat Cattin ira jusqu’à s'absoudre de toute responsabilité, car il retournera contre la gauche toute accusation dans un discours qu'il prononcera à Milan après la fuite de son fils : il rappellera alors que les racines de la violence terroriste ne se trouvaient pas dans sa propre histoire familiale, mais dans l'idéologie du marxisme-léninisme.

En fait, pour Stajano, les destins de ces deux hommes sont tellement liés qu'il est impossible de les dissocier. Le choix de la terreur par le fils est en quelque sorte permis par la protection que son père, ou simplement le nom de son père, lui assure. C'est encore une fois une histoire de servilité, de flatterie, d'opportunisme, d'intérêt personnel placé avant l'éthique publique ; c'est encore une fois un miroir des faiblesses d'une classe dirigeante et de l'arrogance de son pouvoir. Mais la faiblesse des pères n'excuse en rien la génération des fils et n'en fait ni les victimes d'un système laxiste ni des héros de la rédemption des nouvelles générations. Les terroristes de Prima Linea restent pour Stajano des enfants gâtés de la bourgeoisie et de la petite bourgeoisie turinoise, des assassins sans programme, sans un véritable plan, coupés de tout, perdus dans le vide de leur langage auto-référentiel. Stajano restitue avec précision, et grâce à la richesse de sa documentation, les étapes de la dérive vers la lutte armée de ces groupes de jeunes désemparés devant l'éclatement des mouvements et des partis d'extrême gauche au milieu des années 1970. Mais si tout cela aide à comprendre, cela n'en devient pas pour autant un principe de justification, et encore moins d'absolution. Donat Cattin et les siens restent pour Stajano les fils médiocres et irresponsables d'une bourgeoisie qui en devient le complice par opportunisme et par faiblesse.

Ce qui fait la force du livre, c'est le choix conscient et cohérent de création d'un genre de roman-enquête, où la fictionalisation du récit se réalise par l'apport de techniques de narrations très diversifiées tels que le collage 
de documents, la multiplication des points de vue et des niveaux du récit, et l'irruption contrôlée de la subjectivité dans la narration : techniques qui ont pour but de restituer toute la complexité d'un phénomène comme le terrorisme dans ses dimensions à la fois politiques, sociales, culturelles et personnelles. Dans le cas précis de L'Italia nichilista, ce choix d'écriture est parfaitement adapté à la force de l'indignation et de la condamnation morale qui forment comme la trame de l'enquête. C'est peut-être la réponse à la question qui se pose sur la continuité entre la guerre civile et les années de plomb. Il est vrai que Stajano, pour sa part, ne fait aucune allusion à un lien quelconque entre les deux phénomènes. Une fois seulement, quand il tente de qualifier cette sorte d'esthétisme décadent qui est sous-jacent au choix de la terreur de la part des jeunes Turinois, il évoque ses racines dannunziane, son terribilismo petit-bourgeois, pour reprendre une définition de Carlo Ginzburg ${ }^{12}$. Mais pour le reste, la continuité se trouve plutôt dans l'indignation qui s'exprime aussi bien face au fascisme que face au terrorisme, ou aux responsabilités d'une classe dirigeante faible, opportuniste et corrompue, qui paraît reproduire en permanence les mêmes défauts : les généraux se rendent sans combattre pour sauver leur peau, les notables se livrent au fascisme pourvu qu'il défende leurs intérêts, et si le ministre essaie d'épargner la prison à son fils, c'est plus pour sauver sa carrière politique que poussé par ses sentiments paternels. C'est ainsi que Stajano aboutit, dans les deux cas, à la même conclusion : un sentiment d'étrangeté, une prise de distance face à son pays qui, par excès d'amour, le fait rentrer dans la riche galerie bien nourrie des " antiItaliens ».

C'est pour tout cela, grâce à la place qu'il occupe dans l'histoire intellectuelle de l'Italie des trente dernières années, par la force et l'exemplarité de son engagement politique et moral, par sa capacité à créer un genre narratif qui depuis a fait beaucoup d'adeptes et a été tellement imité, que Stajano devrait se voir reconnaître une place importante dans la production littéraire italienne de la seconde moitié du $\mathrm{xx}^{\mathrm{e}}$ siècle.

12. Nous avons nous-même assisté aux cours de Carlo Ginzburg, au printemps 1977 à Bologne. Commentant les menaces proférées par des "autonomi " contre un professeur aveugle (qui refusait de suspendre ses cours pour leur permettre de se réunir en assemblée), Carlo Ginzburg les qualifia justement de prototypes du " terribilismo piccolo borghese ». 